\title{
Study on a New Fault Diagnosis Method Based on Combining Intelligent Technologies
}

\author{
Shiwei $\mathrm{Li}^{1}$ and Di Zhou ${ }^{2}$ \\ ${ }^{1}$ School of Electronic and Information Engineering, Lanzhou Jiaotong University, \\ Lanzhou, 730070, China \\ ${ }^{2}$ Sichuan University of Arts and Science, Dazhou, 635000 China \\ E-mail:zhoudi1981@163.com
}

\begin{abstract}
In allusion to more indeterminate information and higher speed request characteristic in fault diagnosis system, according to the intelligence complementary strategy, a new fault diagnosis(SWPSO-BPN) model based on combining improved particle swarm optimization (PSO) algorithm and Back-propagation $(B P)$ neural network is proposed in this paper. In the SWPSO-BPN method, an improved PSO (SWPSO) algorithm is proposed to optimize the parameters of BP neural network in order to overcome the shortcomings of slow learning speed and being easy to fall into local minimum, and obtain the optimal values of parameter combination in the BP neural network(SWPSO$B P N)$ model. Then proposed SWPSO-BPN model is applied to diagnose the fault in order to obtain a new fault diagnosis (SWPSO-BPN-FD) method. Finally, the proposed SWPSO-BPN-FD method is used to test the data from bearing data center of CWRU. The experimental results show that the proposed SWPSO-BPN-FD method can accurately and effectively realize high precision fault diagnosis of rolling bearing. And this method takes on strong robustness and generalization ability.
\end{abstract}

Keywords: particle swarm optimization; BP neural network; fault diagnosis; optimization; rolling bearing

\section{Introduction}

The bearing is a key mechanical component, it is widely applied to the rotating machinery equipment. The bearing operation state can directly affect the performance of machine. The rolling bearing is the spindle support of motor, it is one of the important component of motor. The bearing fault probability of motor is about $40 \%$. Due to the long-term working of contact stress, the bearing will cause bearing fatigue, crack, indentation and so on, which lead to the abnormal vibration and operation of motor [1]. This abnormal vibration exceeds the allowable value of conventional vibration, it will bring serious damages to the electric power production and personal safety, broken machine and outage. It is thus clear that the normal operation of bearing has a major impact for motor. So the bearing fault diagnosis has received wide attentions and hands, and scientific and technical researchers have carried out a lot of research work. Many methods are proposed in recent decades. Wei et al. [2] proposed a new type of fault decision model based on the information fusion technique. The method used an advanced PSO-BP (particle swarm optimization-back propagation) algorithm to train the neural network and applying the D-S evidential reasoning. Sun et al. [3] proposed an efficient BP-ALM (BP with Adaptive Learning Rate and Momentum coefficient) algorithm to reduce the training time and avoid being trapped into local minima. Then a system of transformer fault diagnosis based on Dissolved Gases Analysis (DGA) with a BP-ALM algorithm is proposed. Sahin et al. [4] proposed a fault diagnosis system for airplane engines using Bayesian networks (BN) and distributed particle swarm optimization (PSO). 
Li et al. [5] proposed a fault diagnosis of nonlinear systems based on intelligent optimization algorithm (PSOSA). The PSOSA could avoid premature convergence of standard particle swarm optimization (PSO) by introducing the probabilistic jumping property of simulated annealing (SA). Nikranjbar et al. [6] proposed a model-based mixed-eccentricity fault detection and diagnosis for induction motors. The proposed algorithm can successfully detect faults and their severity using stator currents. Huang and $\mathrm{Gu}$ [7] proposed an integrating binary particle swarm algorithm into cultural algorithm frame to develop a more efficient cultural binary particle swarm algorithm (CBPSOA) for fault feature selection. Zhang and $\mathrm{Su}$ [8] proposed a turbo-generator vibration faults diagnosis based on particle swarm optimization, BP learning algorithm and rough set theory. Wang et al. [9] proposed an electro-hydraulic regulating system of steam turbine and fault diagnosis based on particle swarm optimization (PSO) and BP neural network. $\mathrm{Wu}$ [10] proposed a robust wavelet v-support vector classifier machine (RWv-SVC) based on wavelet kernel function, robust loss function, PSO and support vector machine. Then a novel fuzzy robust wavelet support vector classifier (FRWSVC) based on a wavelet base function is proposed and an adaptive Gaussian particle swarm optimization (AGPSO) algorithm developed to seek the optimal unknown parameter of the FRWSVC. [11]. Du and $\mathrm{Xi}$ [12] proposed a novel fixture fault diagnosis methodology based on improved Particle Swarm Optimization with Simulated Annealing-based selective neural network Ensemble (PSOSAEN) algorithm for on-line identifying the part variation motion patterns triggering the out-of-control signals. Zhao et al. [13] proposed a fault diagnosis of sensor based on chaos particle swarm optimization algorithm and support vector machine. Zheng et al. [14] a multi-class least square support vector machines (LSSVM) classifier based on particle swarm optimization for transformer fault diagnosis. Wang and Shi [15] proposed a novel method for fault diagnosis based on a bare-bones particle swarm optimization algorithm (BBPSO) and support vector machine (SVM) to accurately recognize the stator winding inter-turn short circuit fault of induction motors. Cui et al. [16] proposed a kind of fault diagnosis method based on RBF Neural Network improved by PSO algorithm to improve the ability of fault diagnosis. Xiao and Feng [17] proposed a KLDA method based on maximal class separability for extracting the optimal features of analog fault data sets, Meanwhile, a novel particle swarm optimization (PSO) based algorithm is developed to tune parameters and structures of neural networks jointly. Azadeh et al. [18] proposed a unique flexible algorithm for classifying the condition of centrifugal pump based on support vector machine hyper-parameters optimization and artificial neural networks (ANNs). Sun et al. [19] proposed an enhanced particle swarm optimization (EPSO)-based support vector classifier (SVC) to extract the support vector from databases, in order to diagnose vibration faults in steam turbine-generator sets (STGS). Dong et al. [20] proposed a modified multi-sensor information fusion method for hydraulic fault diagnosing system based multi-parallel particle swarm optimization (PSO)-Hopfield neural network and modified Dempster-Shafer (D-S). Du et al. [21] proposed a novel fault diagnosis method PSO-RVM based on relevance vector machines (RVM) with particle swarm optimization (PSO) algorithm for plunger pump in truck crane. Wang et al. [22] proposed a compact $\mathrm{K}$ nearest neighbor $(\mathrm{KNN})$ classifier for identifying faults in a power plant thermal system operating at different load level. Yang et al. [23] proposed a diagnosis model based on support vector machine (SVM) and accelerated particle swarm optimization (APSO) for bearing fault diagnosis. Yuan et al. [24] proposed a fault diagnosis method based on support vector machine and improved particle swarm optimization algorithm for engine misfire. Zhu et al. [25] proposed a roller bearing fault diagnosis method based on hierarchical entropy and support vector machine with particle swarm optimization algorithm. Pu and Wang [26] proposed a fault diagnosis method for large-scale railway maintenance equipment based on Back Propagation (BP) neural network and expert system. Wang et al. [27] proposed a novel nearest prototype classifier to diagnose faults in a power plant. $\mathrm{Su}$ et al. [28] proposed a multi-fault 
diagnosis method for rotating machinery based on orthogonal supervised linear local tangent space alignment (OSLLTSA) and least square support vector machine (LS-SVM) to improve the accuracy of fault diagnosis. Yuwono et al. [29] proposed an unsupervised feature selection approach and its application to AFDD in a HVAC system. Yang et al. [30] proposed a gear fault diagnosis based on the EEMD, the kernel function, support vector machine and artificial bee colony algorithm.

For the limitations of the PSO algorithm, the adaptive adjustment strategy of inertia weight $(w)$ is used to improve the PSO algorithm in order to obtain an improved PSO (SWPSO) algorithm. Then the SWPSO algorithm is used to optimize the parameters of BP neural network in order to overcome the shortcomings of slow learning speed and being easy to fall into local minimum, and obtain the optimal values of parameter combination in the BP neural network (SWPSO-BPN) model. Then proposed SWPSOBPN model is applied to diagnose the fault in order to obtain a new fault diagnosis (SWPSO-BPN-FD) method. Finally, the data from bearing data center of Case Western Reserve University (CWRU) is selected to test the performance of SWPSO-BPN-FD method.

The rest of this paper is organized as follows. Section 2 briefly introduces the PSO algorithm and BP neural network. Section 3 proposed an improved PCO algorithm based on the adaptive adjustment strategy of inertia weight. Section 4 proposed an optimized BP neural network based on ASWPSO algorithm. The steps of proposed ASWPSO-BPN are described in this section. Section 5 applies the SWPSO-BPN-FD method to test the data from bearing data center of Case Western Reserve University (CWRU). Finally, the conclusions are discussed in Section 6.

\section{The PSO Algorithm and BP Neural Network}

\subsection{Particle Swarm Optimization (PSO) Algorithm}

Particle swarm optimization (PSO) was originally designed and developed by simulating the social behavior. The PSO is a population-based search algorithm. The PSO consists of many individuals, each individual has a position and a velocity. It works by attracting the particles to search space positions of the high fitness. In the PSO, each particle has a memory function and adjusts its trajectory according to the best visited position and the global best position in the population. The position of best fitness value visited by the swarm is called the global best $(g b)$ and the position of best fitness value by individual is called the local best ( $p b$ ). In a D-dimension space, each particle is treated as a point. The best previous position of the particle in the population is described as $x_{i}=\left(x_{i 1}, x_{i 2}, \cdots, x_{i D}\right)^{T}$.The velocity for particle $i$ is represented as $v_{i}=\left(v_{i 1}, v_{i 2}, \cdots, v_{i D}\right)^{T}$. The velocity and position are updated:

$$
\begin{gathered}
v_{i j}(t+1)=v w_{i j}(t)+c_{1} r_{1}\left(p b_{i j}(t)-x_{i j}(t)\right)+c_{2} r_{2}\left(g b_{i j}(t)-x_{i j}(t)\right) \\
x_{i j}(t+1)=x_{i j}(t)+v_{i j}(t+1)
\end{gathered}
$$

$v_{i j}(t+1)$ is the velocity of particle ${ }^{i}$ at iterations $j, x_{i j}(t+1)$ is position of particle ${ }^{i}$ at iterations ${ }^{j} . w$ denotes the inertia weight coefficient of velocity, ${ }^{c}{ }_{1}$ and $c_{2}$ denote acceleration coefficient. $r_{1}$ and $r_{2}$ are random numbers uniformly distributed in [0,1] which denote remembrance ability. The basic flow of basic PSO algorithm is shown in Figure 1. 


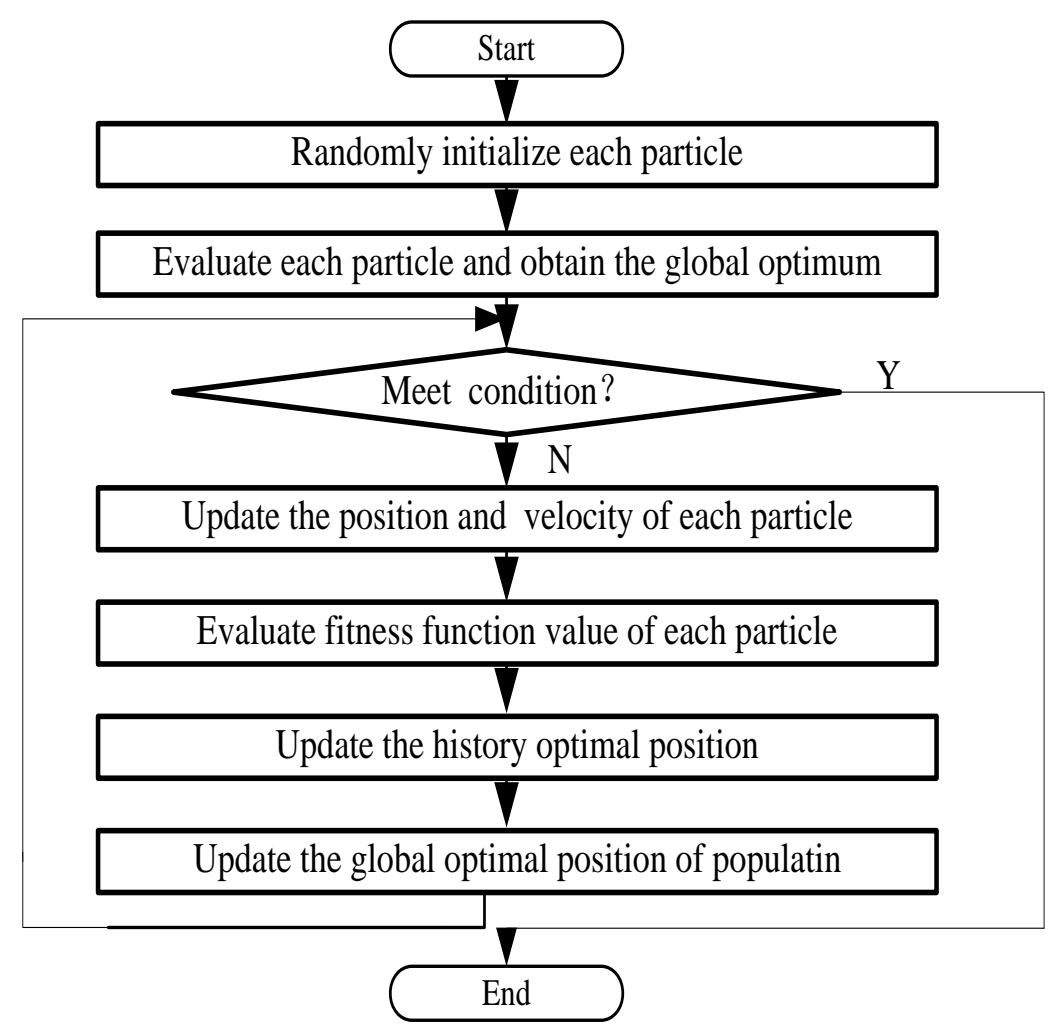

Figure 1. The Basic Flow of PSO Algorithm

\subsection{BP Neural Network}

Neural network is composed of input layer, hidden layer and output layer. BP neural network a multilayer feed-forward neural network. It can store a large amount of the mapping relationships between the input and output and realize the arbitrary nonlinear mapping from the input to output. The BP neural network uses the most fast descent learning rule to constantly adjust the weights and thresholds of network by the error back propagation in order to make the minimum error square of BP neural network. Therefore, the main characteristics of BP neural network is the forward propagation of signal and back-propagation of error. The forward propagation is to input the sample from the input layer, which is transferred the output layer through the hidden layer. If the difference between the actual output and the expected output error value is too large in BP neural network, the back-propagation of error is executed. The back-propagation of error is to transmit the predicted error to the input layer by the hidden layer. Each layer modifies the weights and thresholds according to error signal to obtain the smaller deference between the predicted output and the desired output of BP neural network. The weights and thresholds are cycle and constantly adjusted by using the forward propagation of signal and back-propagation of error until the output error of network decreases the setting error range or the learning times are achieved. The topological structure of BP neural network is shown in Figure2. 


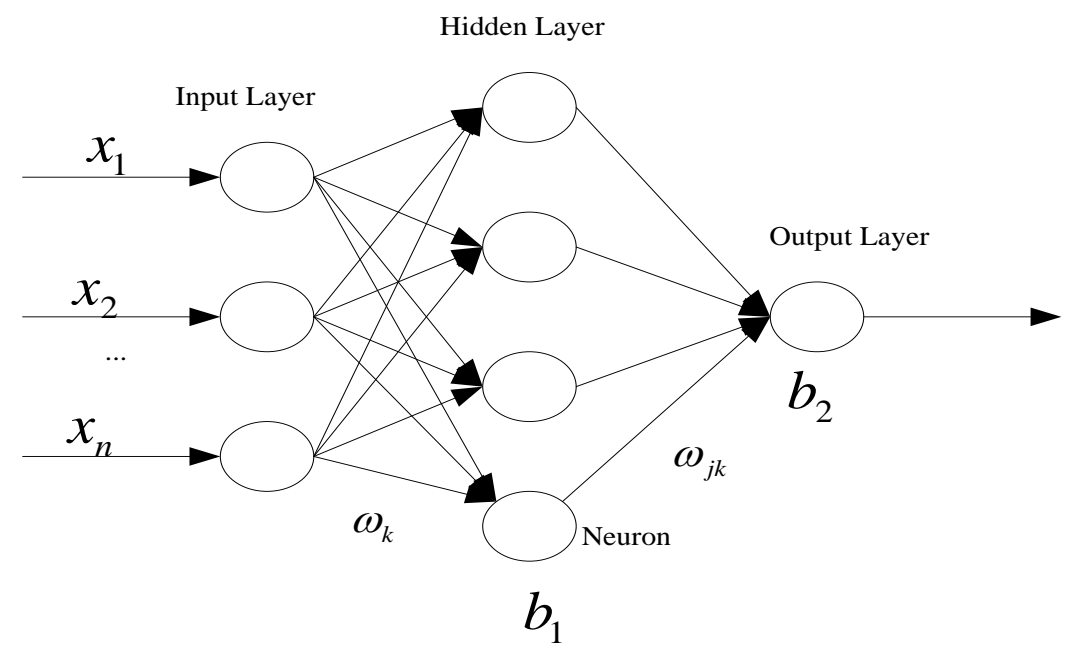

Figure 2. The Topological Structure of Bp Neural Network

Where tan-sigmoid function is used for the neural neurons in the hidden layer. The expression is described as follows:

$$
O=\sum_{i=1}^{n} w_{i} y_{i}
$$

A linear function is used in output layer in order to obtain a broad range values. And there is the $\mathrm{S}$ function of transform function from 0 to 1 , that is to say,

$$
f(x)=\frac{1}{1+e^{-x}}
$$

\section{An Improved PSO (ASWPSO) Algorithm}

In the basic PSO algorithm, the inertia weight $(w)$ is important to judge the convergence speed. The value of inertia weight $(w)$ can keep motion inertia of particle. If the value of inertia weight $(w)$ is larger, it is beneficial to global search and fast convergence speed. But it is difficult to get accurate solution. If the value of inertia weight $(w)$ is smaller, it is beneficial to local search and getting accurate solution. But it exits the slow convergence speed. In the basic PSO algorithm, the value of inertia weight $(w)$ is a constant. In order to obtain better performance of PSO algorithm, dynamic adjustment strategy of inertia weight $(w)$ is used to improve the basic PSO algorithm, and propose an improved PSO (ASWPSO) algorithm in this paper. In the ASWPSO algorithm, the value of inertia weight $(w)$ is larger in the early stage to guarantee the larger search space and avoid premature convergence. With the increasing of iteration, the value of inertia weight $(w)$ is linear reducing to jump out local optimum and improve the convergence accuracy in the later stage. The inertia weight $(w)$ adjustment with linear decreasing is described as follows:

$$
w=w_{\max }-\frac{w_{\max }-w_{\min }}{T_{\max }} \times t
$$

Where $w_{\max }$ and $w_{\min }$ are the maximum value and minimum value of inertia weight ( $w) . T_{\max }$ is the maximum number of iteration, and ${ }^{t}$ is the current number of iteration.

Because the actual process of PSO algorithm is non-linear, the dynamic change of particle is complex. The inertia weight $(w)$ adjustment with linear decreasing can not 
better report the actual optimization process, the convergence speed and accuracy of PSO algorithm are not more satisfying. So an adaptive adjustment strategy of inertia weight $(w)$ is used to keep the population diversity, decrease probability for falling into the local extremum. The adaptive adjustment strategy of inertia weight $(w)$ is described as follows:

$$
w= \begin{cases}w_{\text {min }}+\frac{w_{\text {max }}-w_{\text {min }}}{T_{\text {max }}} \times t & f<\frac{f_{\text {avg }}}{2} \\ w_{\text {min }}+\left(w_{\text {max }}-w_{\text {min }}\right) \times \frac{\frac{f_{\text {avg }}}{2}-f_{\text {min }}}{f-f_{\text {min }}} & \frac{f_{\text {avg }}}{2} \leq f<f_{\text {avg }} \\ w_{\text {max }}-\frac{w_{\text {max }}-w_{\text {min }}}{T_{\text {max }}} \times t & f \geq f_{\text {avg }}\end{cases}
$$

Where $f$ is the current fitness value of particle, $f_{\min }$ is the global minimum fitness value of particle. $f_{\text {avg }}$ the global average fitness value. The strategy uses the current and historical information to improve the updating speed, and better avoid falling into local extremum.

\section{Optimize the BP Neural Network Based on ASWPSO Algorithm}

The weights and thresholds of traditional BP neural network randomly generate. Because the ASWPSO algorithm can obtain more and more smaller (or larger) fitness value, quick convergence speed and more strong global searching ability by continuously updating the position and velocity of particle. So the ASWPSO algorithm is introduced into the BP neural network (ASWPSO-BPN) in order to optimize weights and thresholds and make up the deficiency of BP neural network. In the ASWPSO-BPN, the weights and thresholds of BP neural network is mapped into the particles of population, these parameters are optimized by continuously updating the position and velocity of particle. At the same time, the error mean square value of output in BP neural network is represented by using the fitness value. The goal is to obtain better the initial values of weights and thresholds than the random method and realize the training of BP neural network.

When the BP neural network is trained, a reasonable ASWPSO model is needed to be constructed and the fitness function is determined. The fitness function is the sum of mean squared error square between the actual output value and the desired output value. It is described as follows:

$$
F=\frac{1}{N} \sum_{i=1}^{N} \sum_{j=1}^{O}\left(y_{i j}-p_{i j}\right)
$$

Where $N$ is the number of training samples, $O$ is the number of output neurons, ${ }^{y_{i j}}$ is the expected output value of the $i^{\text {th }}$ sample in the $j^{\text {th }}$ output neuron, $p_{i j}$ is the actual output value of the $i^{\text {th }}$ sample in the $j^{\text {th }}$ output neuron. The steps of proposed ASWPSOBPN are described as follows:

Step1. Initialize the parameters of ASWPSO-BPN 
Population size $(m)$, the number of maximum iteration $\left(T_{\max }\right)$, the maximum velocity $\left(V_{\max }\right)$, the maximum inertia weight $\left(w_{\max }\right)$ and minimum inertia weight $\left(w_{\min }\right)$, the cognition learning factor $\left({ }^{c_{1}}\right)$ and social learning factor $\left({ }^{c_{2}}\right)$, the velocity and position of each particle is initialized.

Step 2. Determine the topological structure of BP neural network according to the practical solving problem. Construct the initial network model and calculate the dimension number of particle.

Step 3. Input the training samples. The initial position vector is taken as the current global optimal value to be mapped into the weights and thresholds. Calculate the actual output value and error mean square value. Then the error mean square value is regarded as fitness function of population.

Step 4. The individual with the weights and thresholds of BP neural network is decoded. The fitness value is calculated according to the structure of the BP network and fitness function. The individual is evaluated.

Step 5. The velocity and position of particle are updated according to the update formula of population. And the maximum speed is limited in the given speed range.

Step 6. For the iterative calculation, the current optimal fitness value is compared with the local optimal value ( $p B$ ) of particle. If the current optimal fitness value is better than the local optimal value ( $p B$ ), then the local optimal value $(p B)$ is replaced by the current optimal fitness value. The local optimal value $(p B)$ of population is updated.

Step 7. The current global optimal value is compared with the global optimal value $(g B)$ of particle. If the current global optimal value is better than the global optimal value $(g B)$, then the global optimal value $(g B)$ is replaced by the current global optimal value. The global optimal value ( $g B$ ) of population is updated.

Step 8. Determine whether the termination condition is satisfied (the error meets the condition or the maximum number of iterations is achieved). If it is true, go the next step. Otherwise, return to Step3.

Step 9. Output and decode the global optimal value $(g B)$. Then the position vector in the global optimal particle is mapped into the weights and thresholds of BP neural network.

Step 10. The test samples are input into the optimized BP neural network (ASWPSOBPN). And the output results of test samples are analyzed and the performance of ASWPSO-BPN is tested.

\section{Fault Diagnosis Case Based on ASWPSO-BPN}

In this paper, the fault data from bearing data center of Case Western Reserve University (CWRU) is selected to verify the effectiveness of proposed SWPSO-BPN-FD method. The inner ring fault, outer ring fault, rolling element fault and normal state are analyzed. The experiment system consists of three-phase AC motor, torque sensor, dynamometer and electronic control device. The bearing of motor is supported by the test bearing. The single local fault is designed by using discharge machining technology in the test bearing. The fault diameter respectively are $0.1778 \mathrm{~mm} 0.3556 \mathrm{~mm}$ and $0.5334 \mathrm{~mm}$, the depth is $0.2794 \mathrm{~mm}$. The 6205-2RS JEM SKF deep groove ball bearing is employed in the experiment. The environments are followed: the Pentium CPU 2.40GHz, 4.0GB RAM, Matlab2010b. Because the initial values of parameters of SWPSO-BPN-FD method could seriously affect the diagnosis result, the most reasonable initial values of parameters are obtained by testing and modifying. The obtained initial values of these 
parameters are: population size $m=100$, the number of maximum iteration $T_{\max }=500$, the maximum velocity $V_{\max }=5.0$, the maximum inertia weight $w_{\max }=0.95$ and minimum inertia weight $w_{\text {min }}=0.5$,the cognition learning factor $\left(c_{1}=2.0\right.$ ) and social learning factor $\left(c_{2}=2.0\right.$ ). For BP neural network, the transfer functions of hidden layer and output layer use $\tan ^{\tan }$, learning function uses leamgd, the training function uses $t$ min $g d$, learning rate is 0.05 , the training error is 0.01 .

The vibration signal is collected by the acceleration sensor and the sampling frequency is $48 \mathrm{kHz}$. Under different rotating speeds of motor, the vibration acceleration signal data of different fault types are the inner ring fault, outer ring fault, rolling element fault and normal state, there are 40 samples. According to the same load/rotating speed and same fault, the 40 samples are divided into 12 data sets. Each set is composed of samples with 4 kinds of different fault types under same load/rotating speed and the diameter of same fault point. The describing of data is shown Tabl.1. The $N$ expresses the normal state, $I$ expresses the inner ring fault, $B$ expresses the rolling element fault, $O$ expresses the outer ring fault.

Table 1. The Describing of Samples

\begin{tabular}{|c|c|c|c|c|c|c|c|c|}
\hline \multirow{2}{*}{ Index } & \multirow{2}{*}{ Data name } & \multirow{2}{*}{ Samples } & \multirow{2}{*}{$\begin{array}{c}\text { Rotating } \\
\text { speed } \\
(r / \mathrm{min})\end{array}$} & \multirow{2}{*}{$\begin{array}{c}\text { Fault } \\
\text { diameter } \\
(m m)\end{array}$} & \multicolumn{4}{|c|}{ Test samples } \\
\hline & & & & & $N$ & $I$ & $B$ & $O$ \\
\hline 1 & $D 007 \_0$ & 20 & 1797 & 0.1778 & 218 & 218 & 219 & 217 \\
\hline 2 & $D 014_{-} 0$ & 20 & 1797 & 0.3556 & 218 & 42 & 223 & 219 \\
\hline 3 & $D 021_{-} 0$ & 20 & 1797 & 0.5334 & 218 & 218 & 218 & 220 \\
\hline 4 & $D 007_{-} 1$ & 20 & 1772 & 0.1778 & 452 & 454 & 455 & 455 \\
\hline 5 & $D 014$ _1 & 20 & 1772 & 0.3556 & 452 & 352 & 454 & 453 \\
\hline 6 & $D 021_{-} 1$ & 20 & 1772 & 0.5334 & 452 & 453 & 455 & 457 \\
\hline 7 & $D 007 \_2$ & 20 & 1750 & 0.1778 & 453 & 454 & 455 & 455 \\
\hline 8 & $D 014 \_2$ & 20 & 1750 & 0.3556 & 453 & 456 & 455 & 455 \\
\hline 9 & $D 021_{-} 2$ & 20 & 1750 & 0.5334 & 453 & 459 & 455 & 456 \\
\hline 10 & $D 007 \_3$ & 20 & 1730 & 0.1778 & 454 & 454 & 457 & 456 \\
\hline 11 & $D 014$ _3 & 20 & 1730 & 0.3556 & 454 & 453 & 455 & 457 \\
\hline 12 & $D 021 \_3$ & 20 & 1730 & 0.5334 & 454 & 457 & 455 & 457 \\
\hline
\end{tabular}

The training of BP neural network is to adjust the weights of each layer. When the BP neural network is trained, a diagnosis model (SWPSO-BPN-FD) is obtained. Then the SWPSO-BPN-FD method is used to identify the rolling bearing fault. The simulation experiment result is shown Table2. 
Table 2. The Simulation Experiment Result

\begin{tabular}{|c|c|c|c|c|c|c|}
\hline \multirow{2}{*}{ Index } & \multirow{2}{*}{ Data name } & \multicolumn{5}{|c|}{ Diagnostic rate of test samples(\%) } \\
\cline { 3 - 7 } & & $N$ & $I$ & $B$ & $O$ & Overall \\
\hline 1 & D007_0 & 100 & 100 & 100 & 99.2 & $\mathbf{9 9 . 8}$ \\
\hline 2 & D014_0 & 99.7 & 93.1 & 97.8 & 100 & 97.7 \\
\hline 3 & D021_0 & 100 & 98.2 & 93.2 & 100 & 97.9 \\
\hline 4 & $D 007 \_1$ & 100 & 100 & 98.5 & 99.6 & $\mathbf{9 9 . 5}$ \\
\hline 5 & $D 014 \_1$ & 100 & 89.4 & 92.8 & 99.9 & 95.5 \\
\hline 6 & $D 021 \_1$ & 100 & 98.3 & 94.2 & 99.8 & $\mathbf{9 8 . 1}$ \\
\hline 7 & $D 007 \_2$ & 100 & 100 & 100 & 100 & $\mathbf{1 0 0}$ \\
\hline 8 & $D 014 \_2$ & 100 & 95.0 & 75.4 & 91.2 & 90.4 \\
\hline 9 & $D 021 \_2$ & 100 & 98.9 & 88.8 & 99.3 & 96.8 \\
\hline 10 & $D 007 \_3$ & 100 & 100 & 100 & 100 & $\mathbf{1 0 0}$ \\
\hline 11 & $D 014 \_3$ & 100 & 81.2 & 78.3 & 99.6 & 89.8 \\
\hline 12 & $D 021 \_3$ & 100 & 99.8 & 90.3 & 93.3 & 95.9 \\
\hline
\end{tabular}

As can be seen from Table2, for the fault data from bearing data center of Case Western Reserve University (CWRU), the proposed SWPSO-BPN-FD method can obtain more higher diagnostic rate. For the data of $D 007_{-}{ }^{2}$ and $D 007 \_3$, the diagnostic rates of inner ring fault, outer ring fault, rolling element fault, normal state and overall are $100 \%$. For the data of $D 007_{-} 0, D 007_{-}{ }^{1}$ and $D 021_{-}{ }^{1}$, the diagnostic rate of overall are respective $99.8 \%, 99.5 \%$ and $98.1 \%$. The experiment results show that the improved PSO algorithm can better obtain the global optimal parameters of BP neural network. So the proposed SWPSO-BPN-FD method has higher fault diagnosis accuracy for the bearing, it is a new method for fault diagnosis.

\section{Conclusion}

The neural network has become an important method for fault diagnosis due to the characteristics of self-learning. The PSO algorithm is a stochastic optimization algorithm, it has characteristics of easy describing, convenient realizing, less adjustment parameters, relatively smaller swarm, less evaluation function times for the convergence, fast convergence speed, parallel processing and good robustness and so on. But when it encountered search space of local minimal points, it also will show its deficiencies, especially when particles are near to local optimal solution in the space, the search efficiency may suddenly reduced. So the adaptive adjustment strategy of inertia weigh is used to improve the PSO algorithm in order to obtain an improved PSO (SWPSO) algorithm. Then the SWPSO algorithm is used to optimize the parameters of BP neural network in order to overcome the shortcomings of slow learning speed and being easy to fall into local minimum, and obtain the optimal values of parameter combination in the BP neural network (SWPSO-BPN) model. It overcomes the shortcomings of the conventional BP neural network. The proposed SWPSO-BPN model is applied to 
diagnose the fault in order to obtain a new fault diagnosis (SWPSO-BPN-FD) method. The SWPSO-BPN-FD method is used to identify the inner ring fault, outer ring fault, rolling element fault, normal state of motor bearing. The experiment results show that the SWPSO-BPN-FD method can efficiently and accurately identify the fault mode of motor bearing. It can be better applied to the fault diagnosis of motor.

\section{Acknowledgements}

This research was supported by the National Natural Science Foundation of China (51475065, U1433124), the Science and Technology Program of Dazhou (Research on the key technology of intelligent fault diagnosis for spindle system of large and middle NCM), China, the Open Project Program of State Key Laboratory of Mechanical Transmissions (Chongqing University) (SKLMT-KFKT-201416), the Open Project Program of the Traction Power State Key Laboratory of Southwest Jiaotong University (TPL1403). The program for the initialization, study, training, and simulation of the proposed algorithm in this article was written with the tool-box of MATLAB 2010b produced by the Math-Works.

\section{References}

[1] W. Deng, X. H. Yang, J. J. Liu, H. M. Zhao, Z. G. Li and X. L. Yan, "A novel fault analysis and diagnosis method based on combining computational intelligence methods", Proceedings of the Institution of Mechanical Engineers, Part I: Journal of Process Mechanical Engineering, vol. 227, no. 3, (2013), pp. 198-210.

[2] X. Wei, N. Q. Shu, P. C. Cui and B. Wu, "Power transformer fault integrated diagnosis based on improved PSO-BP neural networks and D-S evidential reasoning", Automation of Electric Power Systems, vol. 30, no. 7, (2006), pp. 46-50.

[3] Y. J. Sun, S. Zhang, C. X. Miao and J. M. Li, "Improved BP Neural Network for Transformer Fault Diagnosis", Journal of China University of Mining and Technology, vol. 17, no. 1, (2007), pp. 138-142.

[4] F. Sahin, M. C. Yavuz, Z. Arnavut and O. Uluyol, "Fault diagnosis for airplane engines using Bayesian networks and distributed particle swarm optimization", Parallel Computing, vol. 33, no. 2, (2007), pp. 124-143.

[5] L. L. Li, D. H. Zhou and L. Wang, "Fault diagnosis of nonlinear systems based on hybrid PSOSA optimization algorithm", International Journal of Automation and Computing, vol. 4, no. 2, (2007), pp. 183-188.

[6] A. Nikranjbar, M. Ebrahimi and A. S. Wood, "Model-based fault diagnosis of induction motor eccentricity using particle swarm optimization", Proceedings of the Institution of Mechanical Engineers, Part C: Journal of Mechanical Engineering Science, vol. 223, no. 3, (2009), pp. 607-615.

[7] H. Y. Huang and X. S. Gu, "Cultural binary particle swarm optimization algorithm and its application in fault diagnosis", Journal of Donghua University (English Edition), vol. 26, no. 5, (2009), pp. 474-481.

[8] Y. P. Zhang and H. S. Su, "Turbo-generator vibration fault diagnosis based on PSO-BP neural networks", WSEAS Transactions on Systems and Control, vol. 5, no. 1, (2010), pp. 37-47.

[9] X. Y. Wang, X. X. Li and F. S. Li, "Analysis on oscillation in electro-hydraulic regulating system of steam turbine and fault diagnosis based on PSOBP”, Expert Systems with Applications, vol. 37, no. 5, (2010), pp. 3887-3892.

[10] Q. Wu, "Car assembly line fault diagnosis based on robust wavelet SVC and PSO", Expert Systems with Applications, vol. 37, no. 7, (2010), pp. 5423-5429.

[11] Q. Wu and R. Law, "Complex system fault diagnosis based on a fuzzy robust wavelet support vector classifier and an adaptive Gaussian particle swarm optimization", Information Sciences, vol. 180, no. 23, (2010), pp. 4514-4528.

[12] S. C. Du and L. F. Xi, "Fault diagnosis in assembly processes based on engineering-driven rules and PSOSAEN algorithm", Computers and Industrial Engineering, vol. 60, no. 1, (2011), pp. 77-88.

[13] C. L. Zhao, X. B. Sun, S. L. Sun and T. Jiang, "Fault diagnosis of sensor by chaos particle swarm optimization algorithm and support vector machine", Expert Systems with Applications, vol. 38, no. 8, (2011), pp. 9908-9912.

[14] H. B. Zheng, R. J. Liao, S. Grzybowski and L. J. Yang, "Fault diagnosis of power transformers using multi-class least square support vector machines classifiers with particle swarm optimization", IET Electric Power Applications, vol. 5, no. 9, (2011), pp. 691-696.

[15] P. P. Wang and L. P. Shi, "Inter-turn short circuit fault diagnosis of induction motors using the SVM optimized by bare-bones particle swarm optimization", Journal of Theoretical and Applied Information Technology, vol. 45, no. 2, (2012), pp. 573-578. 
[16] L. Cui, C. Y. Wang and B. S. Yang, "Application of RBF neural network improved by PSO algorithm in fault diagnosis", Journal of Theoretical and Applied Information Technology, vol. 46, no. 1, (2012), pp. 268-273.

[17] Y. Q. Xiao and L. G. Feng, “A novel neural-network approach of analog fault diagnosis based on kernel discriminant analysis and particle swarm optimization", Applied Soft Computing Journal, vol. 12, no. 2, (2012), pp. 904-920.

[18] A. Azadeh, M. Saberi, A. Kazem, V. Ebrahimipour, A. Nourmohammadzadeh and Z. Saberi, "A flexible algorithm for fault diagnosis in a centrifugal pump with corrupted data and noise based on ANN and support vector machine with hyper-parameters optimization", Applied Soft Computing Journal, vol. 13, no. 3, (2013), pp. 1478-1485.

[19] H. C. Sun, C. M. Huang and Y. C. Huang, "Fault diagnosis of steam turbine-generator sets using an EPSO-based support vector classifier", IEEE Transactions on Energy Conversion, vol. 28, no. 1, (2013), pp. 164-171.

[20] Z. S. Dong, X. J. Zhang and J. C. Zeng, "Fault diagnosis for hydraulic system on a modified multisensor information fusion method", International Journal of Modelling, Identification and Control, vol. 18, no. 1, (2013), pp. 34-40.

[21] W. L. Du, A. S. Li, P. F. Ye and C. L. Liu, "Fault diagnosis of plunger pump in truck crane based on relevance vector machine with particle swarm optimization algorithm", Shock and Vibration, vol. 20, no. 4, (2013), pp. 781-792.

[22] X. X. Wang, L. Y. Ma and T. Wang, "Hybrid PSO based K nearest neighbor classifier for intelligent fault diagnosis", International Journal of Applied Mathematics and Statistics, vol. 48, no. 18, (2013), pp. 550-558.

[23] G. C. Yang, Q. P. Jian and H. Zhou, "Research on bearing fault diagnosis using APSO-SVM method", Sensors and Transducers, vol. 175, no. 7, (2014), pp. 207-213.

[24] R. D. Yuan, D. Peng, H. Z. Feng and M. Hu, "Fault diagnosis for engine by support vector machine and improved particle swarm optimization algorithm", Journal of Information and Computational Science, vol. 11, no. 13, (2014), pp. 4827-4835.

[25] K. H. Zhu, X. G. Song and D. X. Xue, "A roller bearing fault diagnosis method based on hierarchical entropy and support vector machine with particle swarm optimization algorithm. Measurement", Journal of the International Measurement Confederation, vol. 47, no. 1, (2014), pp. 669-675.

[26] M. Pu and H. R. Wang, "Fault diagnosis expert system for large-scale railway maintenance equipment based on BP neural network", Computer Modelling and New Technologies, vol. 18, no. 11, (2014), pp. 1068-1072.

[27] X. X. Wang, L. Y. Ma and T. Wang, "An optimized nearest prototype classifier for power plant fault diagnosis using hybrid particle swarm optimization algorithm", International Journal of Electrical Power and Energy Systems, vol. 58, no. 6, (2014), pp. 257-265.

[28] Z. Q. Su, B. P. Tang, Z. R. Liu and Y. Qin, "Multi-fault diagnosis for rotating machinery based on orthogonal supervised linear local tangent space alignment and least square support vector machine", Neurocomputing, vol. 157, no. 6, (2015), pp. 208-222.

[29] M. Yuwono, Y. Guo, J. Wall, J. M. Li, S. West, G. Platt and S.W. Su, "Unsupervised feature selection using swarm intelligence and consensus clustering for automatic fault detection and diagnosis in Heating Ventilation and Air Conditioning systems", Applied Soft Computing Journal, vol. 34, no. 6, (2015), pp. 402-425.

[30] D. L. Yang, Y. L. Liu, S. B. Li, X. J. Li and L. Y. Ma, "Gear fault diagnosis based on support vector machine optimized by artificial bee colony algorithm", Mechanism and Machine Theory, vol. 90, no. 8, (2015), pp. 219-229. 


\section{Authors}

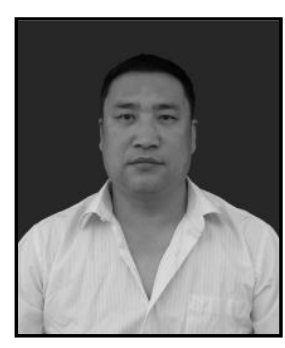

Shiwei Li, He received the Master degree in computer application from Lanzhou University in 2005, Lanzhou, China. The main research directions: fault detection of oil well, nondestructive testing, development technology of Industrial control software.

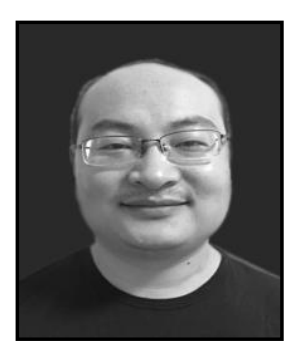

Di Zhou, Associate research fellow, received the Master degree in software engineering from University of Electronic Science and Technology of China in 2009, Chengdu, China. The main research directions: Artificial intelligence. 